Arch. Tierz., Dummerstorf 43 (2000) 5, 421-430

Aus dem Institut fur Tierzucht und Tierhaltung mit Tierklinik der Martin-Luther-Universitat Halle-Wittenberg

NORBERT MIELENZ und LUTZ SCHÜLER

\title{
Langzeitselektion bei asymmetrischer Merkmalsverteilung
}

\author{
Herrn Professor Dr. Dr. h.c. Gerhard von Lengerken zum 65. Geburtstag gewidmet
}

\begin{abstract}
Summary
Title of the paper: Long-term selection by asymmetric trait distribution

A quantitative trait is assumed to be genetically affected by a polygenic effect and a major effect of a single dialellic locus. Such a model is called mixed model of inheritance and the identified gene is denoted as quantitative trait locus (QTL). By choosing the part of the QTL-variance, the degree of dominance and the frequency of the favourable allele it is possible to generate distributions with given level of asymmetry characterised by skewness and kurtosis. If the ratio of QTL- and phenotypic variance is small (less than 8\%), then genotype-environment interaction can be used in the mixed inheritance model to explain values of skewness and kurtosis estimated with poultry data. The situation is considered where the environmental variances given for the three QTL-genotypes show a wide range. In most of these cases the short- and long-term selection does not effects the high values for skewness and kurtosis over multiple generations. Further the ratio of response to selection and the difference of selection depends on the selection intensity.
\end{abstract}

Key Words: mixed inheritance model, long-term response to selection, non-normality

\section{Zusammenfassung}

Zur Erzeugung asymmetrischer Verteilungen wird angenommen, dass die genetische Fundierung eines Merkmals auf einem identifizierten Einzelgen und einer polygenen Komponente beruht. Ein derartiges Modell wird als gemischtes Modell der Vererbung bezeichnet. Der identifizierte Genort heißt „Quantitative Trait Locus“ (QTL). Durch entsprechende Wahl des Anteils der QTL-Varianz an der phănotypischen Varianz in der Population, des Dominanzgrades und der Frequenz des positiven Allels lassen sich Verteilungen erzeugen, die einem gewissen Grad der Asymmetrie, charakterisiert durch Schiefe und Exzeß, genugen. Ist das Verhăltnis aus QTL-Varianz und phănotypischer Varianz gering (kleiner als $8 \%$ ), so muß im gemischten Modell der Vererbung Genotyp-Umwelt Interaktion zugelassen werden, um Werte für Schiefe und Exzeß, geschătzt an Geflugeldaten, erklären zukönnen. Es wird der Fall betrachtet, dass die Umweltvarianzen am QTL variieren. In dieser Situation stabilisieren sich die Werte von Schiefe und Exzeß auch unter den Einfluß von mittel- und langfristiger Selektion auf hohem Niveau. Weiterhin zeigt der Quotient aus Selektionserfolg und Selektionsdifferenz eine deutliche Abhăngigkeit vom Anteil der selektierten Tiere in der Population.

Schlusselworter: Gemischtes Vererbungsmodell, Langzeitselektion, Nicht-Normalverteilung

\section{Einleitung}

Die Vorhersage der genetischen Veränderung eines Merkmals infolge von Selektionsmaßnahmen ist eine zentrale Aufgabe bei der Einführung und Anwendung neuer Zuchtverfahren. Die klassische Theorie der Indexselektion geht von mehrdimensionaler Normalverteilung für Genotyp und Phänotyp aus. Bei Stutzungsselektion nach Eigenleistung in beiden Geschlechtern besteht, unendliche Populationgröße vorausgesetzt, zwischen Selektionsdifferenz (SD) und Selektionserfolg (SE) unabhängig von der Remontierungsrate der Zusammenhang $\mathrm{SE}=\mathrm{h}^{2} \mathrm{SD}$, wobei $\mathrm{h}^{2}$ die Heritabilităt im 
Merkmal $\mathrm{x}$ ist. Obige Formel, mehr oder weniger modifiziert, fand Eingang bei der Vorhersage des mittel- und langfristigen SE unter Beachtung des Bulmer-Effektes (BULMER, 1971) und bei der Untersuchung von Selektionsstrategien, falls neben einer polygenen Komponente ein identifiziertes Einzelgen die Merkmalsausprägung steuert (PONG-WONG und WOOLIAMS, 1998; DEKKERS und VAN ARENDONK 1998). Ein derartiges Modell wird auch als gemischtes Modell der Vererbung (GMV), der herausgehobene Genort als Hauptgenort oder QTL (Quantitative Trait Locus) und die Varianz, verursacht durch das Hauptgen, als QTL-Varianz bezeichnet. Die Dichtefunktion der zugehörigen Merkmalsausprägung erweist sich als Mischung von gewöhnlich 3 Normalverteilungen. Durch Wahl des Anteils der QTL-Varianz an der genetischen Gesamtvarianz, des Dominanzgrades und der Frequenz des gewünschten QTL-Allels lassen sich Verteilungen erzeugen, die einem vorgegebenen Grad der Asymmetrie, charakterisiert durch Schiefe und Exzeß, genügen (MIELENZ und SCHÜLER, 1998). In dieser Arbeit wird untersucht, welchen Einfluß mittel- und langfristige Selektion, nach einem Index aus Phänotyp- und QTL-Information, auf die Entwicklung von Schiefe und Exzeß haben. Um Asymmetrien für den Quotienten aus $\mathrm{SE}$ und SD in Abhängigkeit von der Remontierung erklären zukönnen, müssen zusätzliche Annahmen über die polygenen und Umweltvarianzen innerhalb der verschiedenen QTL-Genotypen getroffen werden.

Der einfachste Fall eines gemischten Modells der Vererbung wird betrachtet, indem angenommen wird, daß die genetische Fundierung eines Merkmals auf einem identifizierten Einzelgen (QTL) und einer polygenen Komponente beruht. Das Modell besitzt die Gestalt:

$$
x_{i j}=\mu_{i}+a_{i j}+u_{i j}
$$

Hierbei sind:

$$
\begin{array}{lll}
x_{i j} & - & \text { Leistung von Tier } j \text { mit Genotyp } i \\
a_{i j} & - & \text { additiv-genetischer Effekt von Tier } j,\left(\operatorname{Var}\left(a_{i j}\right)=\sigma_{a_{i}}^{2}\right) \\
u_{i j} & - & \text { zufälliger Umwelteffekt, }\left(\operatorname{Var}\left(u_{i j}\right)=\sigma_{u_{i}}^{2}\right)
\end{array}
$$

Sei vorausgesetzt, daß am QTL zwei Allele $A_{1}$ und $A_{2}$ vorliegen, wobei das erste Allel die Frequenz $p$ besitze. Weiterhin gelten die folgenden Verteilungsannahmen:

$$
x_{i j} \sim N\left(\mu_{i}, \sigma_{i}^{2}\right) \quad \text { mit } E\left(x_{i j}\right)=\mu_{i} \text { und } \sigma_{i}^{2}=\sigma_{a_{i}}^{2}+\sigma_{u_{i}}^{2}
$$

Im unterstellten Ein-Locus-Fall kann $\mu_{i}$ die drei Ausprägungen $c+a, c+d$ und $c-a$ annehmen. Für die drei möglichen Genotypen $A_{1} A_{1}, A_{1} A_{2}$ und $A_{2} A_{2}$ werden nachfolgend äquivalent die Kurzbezeichnungen $G_{1}, G_{2}$ und $G_{3}$ verwendet. Die zugehörigen Genotypenfrequenzen $P\left(G_{i}\right)$ seien mit $p_{i}$ abgekürzt. Es soll von HardyWeinberg-Gleichgewicht ausgegangen werden, insbesondere seien Einzelgen und polygene Komponente voneinander unabhängig. 
Die Dichtefunktion aller Tiere für das Merkmal x, erklärt durch Modell (1), besitzt die Darstellung:

$$
\begin{aligned}
p(x)= & \sum_{i} p_{i} \cdot \varphi\left(x, \mu_{i}, \sigma_{i}^{2}\right) \\
& \varphi\left(x, \mu_{i}, \sigma_{i}^{2}\right) \quad-\quad \text { Dichte der eindimensionalen Normalverteilung mit }
\end{aligned}
$$$$
\text { Erwartungswert } \mu_{i} \text { und Varianz } \sigma_{i}^{2}
$$

Mit Hilfe obiger Dichte erhält man für das Populationsmittel $\mu$ und die phänotypische Varianz $\sigma_{x}^{2}$ die Darstellung:

$$
\mu=\sum_{i} p_{i} \mu_{i} ; \quad \text { und } \quad \sigma_{x}^{2}=\sum_{i} p_{i}\left(\mu_{i}-\mu\right)^{2}+\sum_{i} p_{i} \sigma_{i}^{2}
$$

Der Anteil $\sum p_{i}\left(\mu_{i}-\mu\right)^{2}$, verursacht durch die Variabilität am Hauptgen, wird nachfolgend als QTL-Varianz $\left(\sigma_{q}^{2}\right)$ bezeichnet.

2.1 Berechnung von Schiefe und Exzeß

Das zentrale Moment 3-ter Ordnung berechnet sich definitionsgemäß wie folgt:

$$
E(x-\mu)^{3}=\sum p_{i} \int_{-\infty}^{\infty}(x-\mu)^{3} \varphi\left(x, \mu_{i}, \sigma_{i}^{2}\right) \cdot d x
$$

Setzt man $d_{i}=\mu_{i}-\mu$, so folgt bei Verwendung der bekannten Momente der standardisierten Normalverteilung (vgl. HAFNER, 1989) für die Schiefe:

$$
\gamma_{1}=\frac{E(x-\mu)^{3}}{\sigma_{x}^{3}}=\frac{\sum_{i} p_{i}\left(3 \sigma_{i}^{2} d_{i}+d_{i}^{3}\right)}{\left(\sum_{i} p_{i}\left(\sigma_{i}^{2}+d_{i}^{2}\right)\right)^{\frac{3}{2}}}
$$

Im Spezialfall $\sigma_{i}^{2}=\sigma^{2}$ ergibt sich die Beziehung $\gamma_{1}=\gamma_{1, q} \cdot\left(s^{2} h^{2}\right)^{\frac{3}{2}}$ mit $s^{2}=\sigma_{q}^{2} / \sigma_{g}^{2}$ und $h^{2}=\sigma_{g}^{2} / \sigma_{x}^{2}$. Das heißt, die Schiefe, verursacht durch den QTL, wird nicht im vollen Umfang auf die phänotypische Merkmalsausprägung übertragen. Die absolute Reduzierung ergibt sich durch Multiplikation mit dem Faktor $\left(s^{2} h^{2}\right)^{\frac{3}{2}}$. Analoge Ableitungen liefern für den Exzeß die Darstellung:

$$
\gamma_{2}=\frac{E(x-\mu)^{4}}{\left(\sigma_{x}^{2}\right)^{2}}-3=\frac{\sum_{i} p_{i}\left\{3 \sigma_{i}^{2}\left(\sigma_{i}^{2}+2 d_{i}^{2}\right)+d_{i}^{4}\right\}}{\left(\sum_{i} p_{i}\left(\sigma_{i}^{2}+d_{i}^{2}\right)\right)^{2}}-3
$$


2.2 Erzeugung asymmetrischer Verteilungen

Von MIELENZ und SCHÜLER (1998) wurde untersucht unter welchen Anforderungen an die Anteile von QTL- und polygener Varianz vorgegebene Werte von Schiefe und Exzeß eingehalten werden.

Folgende generelle Annahmen werden getroffen:

- Unendliche Populationsgröße, Panmixie, Anpaarungsverhältnis 1:1

- Die Heritabilität im weiteren Sinn wird mit 0.4 angesetzt.

- Die Frequenz des gewünschten Allels sei gleich 0.8 .

- Am QTL liegt vollständige Dominanz vor. Es gilt also $a=d$.

- Da Schiefe und Exzeß von einer Standardisierung unbeeinflußt bleiben wird $\mu=0.0$ und $\sigma_{x}^{2}=1.0$ gewählt.

In Abhängigkeit von $s^{2}$, dem Anteil der QTL-Varianz an der genetischen Varianz, ergeben sich die in Tabelle 1 dargestellten Zusammenhänge. Die Werte von $c, a$ und $d$ in Tabelle 1 sind standardisiert und gewährleisten für das Populationsmittel den Wert Null sowie den vorgegeben Anteil an der genetischen Gesamtvarianz erklärt durch die Variation am QTL.

Tabelle 1

Schiefe und Exzeß in Abhăngigkeit von $s^{2}=\sigma_{q}^{2} / \sigma_{g}^{2}$ (Skewness and kurtosis calculated for different ratios of $\sigma_{q}^{2}$ to $\sigma_{g}^{2}$ )

\begin{tabular}{cccccccc}
\hline \multirow{2}{*}{ Nr.: } & \multicolumn{9}{c}{ Populationsparameter } & \multicolumn{1}{c}{$\sigma_{u}^{2}$} & $\gamma_{1}$ & $\gamma_{2}$ \\
\hline 1 & $s^{2}$ & $a=d$ & & $\sigma_{a}^{2}$ & $\sigma_{u}^{2}$ & 0,5131 \\
2 & 0,40 & 1,0206 & $-0,9390$ & 0,24 & 0,60 & $-0,3005$ & 0,1283 \\
3 & 0,20 & 0,7217 & $-0,6640$ & 0,32 & 0,60 & $-0,1062$ & 0,0321 \\
4 & 0,10 & 0,5103 & $-0,4695$ & 0,36 & 0,60 & $-0,0376$ & 0,0080 \\
\hline
\end{tabular}

Die Asymmetriemaße $\gamma_{1}$ und $\gamma_{2}$ in Tabelle 1 sind unter der Annahme identischer polygener und Umweltvarianzen für alle Genotypen berechnet. Zur Nachbildung von Werten für die Schiefe zwischen -2 und -0.5 und für den Exzeß zwischen 2 und 5, wie z.B. in der Legehuhnzucht mit Praxisdaten geschätzt (KOERHUIS, 1996; SAVAS et al., 1998), werden die folgenden Thesen herangezogen (vgl. FALCONER, 1984).

These A: Individuen mit hohem genetischen Niveau können a) weniger und b) stärker durch Umwelteinflüsse beeinflußt werden. Im gemischten Modell der Vererbung gelten für die Umweltvarianzen folglich die Relationen a) $\sigma_{u_{1}}^{2} ; \sigma_{u_{2}}^{2}<\sigma_{u_{3}}^{2}$ und b) $\sigma_{u_{1}}^{2} ; \sigma_{u_{2}}^{2}>\sigma_{u_{3}}^{2}$.

These B: Individuen mit hohem genotypischen Wert am QTL zeigen a) weniger und b) mehr genetische Variation in ihrer polygenen Komponente als solche, die einen niedrigeren genotypischen Wert am QTL besitzen. Es gelten also im GMV für die polygenen Varianzen die Relationen a) $\sigma_{a_{1}}^{2} ; \sigma_{a_{2}}^{2}<\sigma_{a_{3}}^{2}$ und b) $\sigma_{a_{1}}^{2} ; \sigma_{a_{2}}^{2}>\sigma_{a_{3}}^{2}$. 
These A entspricht einer Interaktion zwischen QTL-Genotyp und Umweltkomponente. In den nachfolgenden Untersuchungen wird Konstellation a) zugrunde gelegt. Setzt man Variante b) unter der zusätzlichen Annahme $\sigma_{u_{1}}^{2}>\sigma_{u_{2}}^{2}$ voraus, so werden bei scharfer Remontierung nach wenigen Generationen phänotypischer Selektion die homozygot positiven Tiere vollständig erkannt und folglich eine symmetrische Verteilung erreicht.

Die Konstellationen von These B beschreiben eine Interaktion zwischen den genotypischen Werten am QTL und der polygenen Komponente. Diese Situation könnte aus einer zeitweiligen Interaktion zwischen Genotyp und polygener Komponente resultieren. Eine unterschiedliche selektive Behandlung der Genotypen und die Anpaarung ausschließlich innerhalb gewisser Genotypen wäre als Vorgeschichte für diese Konstellation denkbar.

Unter der Annahme von These A wurden die polygenen Heritabilitäten für Genotyp $G_{1}$ und $G_{2}$ vorgegeben und die Umweltvarianz für den Genotyp $G_{3}$ modifiziert, so daß die generellen Vorgaben gewahrt blieben. Entsprechend wurde unter These B mit der polygenen Varianzkomponente von Genotyp $G_{3}$ verfahren.

Tabelle 2

Schiefe und Exzeß in Abhăngigkeit von $s^{2}=\sigma_{q}^{2} / \sigma_{g}^{2}$ bei Variation der Umweltvarianzen (These A) oder der polygenen Varianzen (These B) (Skewness and kurtosis calculated for different values of $s^{2}=\sigma_{q}^{2} / \sigma_{g}^{2}$ by variation of the enviromental variances (statement A) or of the polygenic variances (statement B)

\begin{tabular}{|c|c|c|c|c|c|c|c|c|c|c|}
\hline \multirow[b]{2}{*}{ These } & \multirow[b]{2}{*}{$s^{2}$} & \multirow[b]{2}{*}{$h_{p_{1}}^{2}$} & \multirow[b]{2}{*}{$h_{p_{2}}^{2}$} & \multirow[b]{2}{*}{$h_{p_{3}}^{2}$} & \multirow[b]{2}{*}{$\sigma_{a}^{2}$} & \multicolumn{3}{|c|}{ Umweltvarianzen } & \multirow[b]{2}{*}{$\gamma_{1}$} & \multirow[b]{2}{*}{$\gamma_{2}$} \\
\hline & & & & & & $\sigma_{u_{1}}^{2}$ & $\sigma_{u_{2}}^{2}$ & $\sigma_{u_{3}}^{2}$ & & \\
\hline Al & 0,40 & 0,50 & 0,30 & 0,03 & 0,24 & 0,24 & 0,56 & 6,68 & $-1,78$ & 10,8 \\
\hline A2 & 0,20 & 0,50 & 0,30 & 0,08 & 0,32 & 0,32 & 0,75 & 3,91 & $-0,68$ & 3,13 \\
\hline A3 & 0,10 & 0,50 & 0,30 & 0,13 & 0,36 & 0,36 & 0,84 & 2,52 & $-0,27$ & 1,08 \\
\hline A4 & 0,05 & 0,50 & 0,30 & 0,17 & 0,38 & 0,38 & 0,89 & 1,83 & $-0,12$ & 0,50 \\
\hline \multirow[b]{2}{*}{ These } & & & & & & \multicolumn{3}{|c|}{ Polygene Varianzen } & & \\
\hline & $s^{2}$ & $h_{p_{1}}^{2}$ & $h_{p_{2}}^{2}$ & $h_{p_{3}}^{2}$ & $\sigma_{u}^{2}$ & $\sigma_{a_{1}}^{2}$ & $\sigma_{a_{2}}^{2}$ & $\sigma_{a_{3}}^{2}$ & $\gamma_{1}$ & $\gamma_{2}$ \\
\hline B1 & 0,40 & 0,30 & 0,20 & 0,53 & 0,60 & 0,26 & 0,15 & 0,69 & $-0,41$ & 0,96 \\
\hline B2 & 0,20 & 0,30 & 0,20 & 0,82 & 0,60 & 0,26 & 0,15 & 2,69 & $-0,52$ & 1,92 \\
\hline B3 & 0,10 & 0,30 & 0,20 & 0,86 & 0,60 & 0,26 & 0,15 & 3,69 & $-0,44$ & 2,19 \\
\hline B4 & 0,05 & 0,30 & 0,20 & 0,87 & 0,60 & 0,26 & 0,15 & 4,19 & $-0,34$ & 2,26 \\
\hline
\end{tabular}

Auf Grund der Ergebnisse aus den Tabellen 1 und 2 lassen sich folgende Schlußfolgerungen ziehen.

- Die Schiefe der Merkmalsausprägung ist abhängig vom Anteil der QTLVarianz an der Gesamtvarianz.

- Liegt der Anteil der QTL-Varianz an der genetischen Gesamtvarianz unter $20 \%$, so wird es bei Varianzhomogenität über alle QTL-Genotypen fast aussichtslos Verteilungen mit großer (negativer) Schiefe zu erzeugen.

- Für die Legeleistung beim Huhn realistische Asymmetrien stellen sich ein, falls z.B. die Umweltvarianzen für jeden Genotyp variieren und die polygenen Varianzen konstant gehalten werden. Durch diese Vorgehensweise lassen sich auch für $s^{2} \leq 20 \%$ Verteilungen mit relevanter negativer Schiefe 
erzeugen, falls die Verhältnisse zwischen den 3 Umweltvarianzen hinreichend weit gestaltet werden.

2.3 Schiefe und Exzeß unter dem Einfluß von Selektion

Der Formelapparat zur Berechnung der genetischen Veränderungen in Modell (1) bei Stutzungsselektion über $\mathrm{n}$ Generationen, ist in der Arbeit von PONG-WONG und WOOLIAMS (1998) zu finden. Bei der Berechnung der polygenen Mittel und Varianzen in der Nachkommengeneration ist zu beachten, daß die einzelnen Elterngenotypen unterschiedliche polygene Erwartungswerte besitzen (vgl. SIMIANER, 1995). Eine ausführliche Darstellung des Formelapparates zur Berechnung der polygenen Mittel und der polygenen Varianzen auf Generation $\mathrm{n}$ bei genereller Stutzungsselektion (inklusive Auf- und Abwärtsselektion) findet man bei MIELENZ (2000). Um den Einfluß von Selektion auf die genetischen Parameter abzuschätzen, wird Stutzungsselektion über 20 Generationen a) nach dem Phänotyp und b) nach einem Index aus genotypischen Werten und Phänotyp bei einer Remontierung $\alpha$ von $20 \%$ in beiden Geschlechtern unterstellt.

Der untersuchte Index wurde mit Hilfe der mittleren polygenen Heritabilität $h_{p}^{2}$ wie folgt gebildet (vgl. SIMIANER, 1995):

$$
I=\mu_{i}+h_{p}^{2}\left(x_{y}-\mu_{i}\right) \quad \text { mit } \quad h_{p}^{2}=0,32 /(0,32+0,6)=0,3478
$$

Die Auswirkungen einer Langzeitselektion unter These A (gemäß Variante A2 aus Tabelle 2) auf die Populationsparameter einschließlich der Asymmetriemaße zeigt Tabelle 3.

Tabelle 3

Entwicklung von Schiefe, Exzeß, Allelfrequenz und kumulativem Selektionserfolg unter These A uber die Generationen bei $s^{2}=0.2$. (changes of skewness, kurtosis, frequency of the favourable allele and cumulated selection response using statement A over the generations by $s^{2}=0.20$.)

\begin{tabular}{ccccccccc}
\hline & \multicolumn{3}{c}{ Phănotypische Selektion } & \multicolumn{5}{c}{ Indexselektion } \\
Generat. & $\gamma_{1}$ & $\gamma_{2}$ & $p\left(A_{1}\right)$ & $S E_{\text {kum }}$ & $\gamma_{1}$ & $\gamma_{2}$ & $p\left(A_{1}\right)$ & $S E_{\text {kum }}$ \\
\hline 1 & $-0,90$ & 3,90 & 0,78 & 0,49 & $-0,81$ & 3,70 & 0,80 & 0,50 \\
2 & $-0,96$ & 4,06 & 0,77 & 0,92 & $-0,80$ & 3,73 & 0,81 & 0,95 \\
3 & $-0,98$ & 4,10 & 0,77 & 1,33 & $-0,78$ & 3,66 & 0,82 & 1,38 \\
4 & $-0,99$ & 4,12 & 0,76 & 1,75 & $-0,75$ & 3,58 & 0,83 & 1,81 \\
5 & $-1,00$ & 4,12 & 0,76 & 2,16 & $-0,72$ & 3,50 & 0,83 & 2,24 \\
10 & $-1,02$ & 4,13 & 0,75 & 4,23 & $-0,64$ & 3,20 & 0,85 & 4,39 \\
20 & $-1,03$ & 4,13 & 0,75 & 8,36 & $-0,58$ & 2,98 & 0,86 & 8,70 \\
\hline
\end{tabular}

Gemäß Tabelle 3 stabilisieren sich Schiefe und Exzeß auf hohem Niveau auch unter dem Einfluß von Selektion. Bei der Stutzungsselektion nach dem Phänotyp kommt es sogar zu einem leichten Abfall für die Frequenz des gewünschten Allels. Dazu gegensätzliche Ergebnisse erhält man, falls die Konstellation von Variante B2 aus Tabelle 2 über 20 Generationen Selektion verfolgt wird. Da Unabhängigkeit zwischen QTL und polygener Komponente vorausgesetzt wurde, reduziert sich bereits auf der ersten Generation der Unterschied zwischen den polygenen Varianzen erheblich. Besitzt ein Nachkomme den Genotyp $G_{k}$ bei elterlichen Genotypen $G_{i}$ und $G_{j}$, so ist 
die Varianz des Mendelian-Sampling für derartige Tiere gleich $0.25 \cdot\left(\sigma_{a_{i}}^{2}+\sigma_{a_{a}}^{2}\right)$. Es liegt die Konstellation von genetischen Gruppen vor, wobei die Anpaarung nicht mehr nur innerhalb, sondern über alle Gruppen erfolgt. Die polygenen Varianzen nivellieren sich, so daß erwartungsgemäß eine Reduzierung der Asymmetrie eintritt.

Tabelle 4

Entwicklung von Schiefe, Exzeß, Allelfrequenz und kumulativem Selektionserfolg unter These B uber die Generationen bei $s^{2}=0,2$ (Changes of skewness, kurtosis, frequency of the favourable allele and cumulated selection response using statement B over the generations by $s^{2}=0.2$ )

\begin{tabular}{ccccccccc}
\hline & \multicolumn{3}{c}{ Phänotypische Selektion } & \multicolumn{4}{c}{ Indexselektion } \\
Generat. & $\gamma_{1}$ & $\gamma_{2}$ & $p\left(A_{1}\right)$ & $S E_{\text {kum }}$ & $\gamma_{1}$ & $\gamma_{2}$ & $p\left(A_{1}\right)$ & $S E_{\text {kum }}$ \\
\hline 1 & $-0,10$ & 0,24 & 0,82 & 0,40 & $-0,10$ & 0,14 & 0,84 & 0,36 \\
2 & $-0,07$ & 0,09 & 0,82 & 0,81 & $-0,06$ & 0,09 & 0,87 & 0,70 \\
3 & $-0,09$ & 0,11 & 0,82 & 1,16 & $-0,05$ & 0,07 & 0,89 & 1,01 \\
4 & $-0,08$ & 0,11 & 0,84 & 1,48 & $-0,04$ & 0,05 & 0,91 & 1,33 \\
5 & $-0,07$ & 0,10 & 0,86 & 1,80 & $-0,03$ & 0,04 & 0,92 & 1,64 \\
10 & $-0,02$ & 0,03 & 0,93 & 3,36 & $-0,01$ & 0,01 & 0,96 & 3,23 \\
20 & $-0,00$ & 0,00 & 0,97 & 5,58 & $-0,00$ & 0,00 & 0,98 & 6,48 \\
\hline
\end{tabular}

Der in Tabelle 4 verwendete Index wichtet QTL-Information und die Schätzung für die polygene Komponente mit einer mittleren polygenen Heritabilität. Da in der Basispopulation je Genotyp extrem unterschiedliche polygene Varianzen vorausgesetzt wurden, bleibt in diesem Spezialfall die praktizierte Indexselektion auf den ersten Generationen im Vergleich zur phänotypischen Selektion hinsichtlich des Selektionserfolges zurück. Auf Grund der Ergebnisse aus Tabelle 3 und 4 lassen sich folgende Schlußfolgerungen ziehen:

- Unter der Annahme von These A (Konstellation a) vergrößern sich Schiefe und Exzeß unter dem Einfluß von Selektion nach einer Generation absolut. Die Asymmetriemaße bleiben über mehrere Generationen Selektion auf annähernd konstantem Niveau.

- Unter der Annahme von These B (Konstellation a), bei Ausschluß von Interaktion zwischen QTL und polygener Komponente, reduzieren sich Schiefe und Exzeß durch eine Generation Selektion erheblich. Nach wenigen Generationen Selektionseinfluß liegt bereits eine vollständig symmetrische Merkmalsverteilung in der Population vor.

\subsection{Realisierte Heritabilität in Abhängigkeit von der Remontierung}

Die generellen Vorgaben aus Abschnitt 2.2 werden beibehalten. Zur Erzeugung asymmetrischer Verteilungen wird vorausgesetzt, daß Tiere mit hohem genotypischen Wert am QTL einer anderen Umweltvariation als solche mit niedrigem genotypischen Wert unterliegen. Die realisierten Heritabilitäten, definiert als Quotient aus Selektionserfolg und Selektionsdifferenz bei Stutzungsselektion nach Eigenleistung in beiden Geschlechtern, in Abhängigkeit von der Remontierung für unterschiedliche Wahl der polygenen Heritabilitäten, enthält Tabelle 5.

Die Ergebnisse aus Tabelle 5 zeigen: Durch Auslenkung der Verhältnisse zwischen den polygenen Heritabilitäten vergrößert sich die Schiefe absolut wobei gleichzeitig 
Tabelle 5

Realisierte Heritabilităt in Abhăngigkeit von der Remontierung bei $s^{2}=0,2$ (polygene Heritabilităt maximal für homozygot positiven Genotyp) (Realized heritability calculated for different selection intensities by $s^{2}=0.2$ )

\begin{tabular}{ccccccccccccccc}
\hline \multirow{1}{*}{ Nr.: } & $h_{p_{1}}^{2}$ & $h_{p_{2}}^{2}$ & $h_{p_{3}}^{2}$ & 0,1 & 0,2 & 0,3 & 0,4 & 0,5 & 0,6 & 0,7 & 0,8 & 0,9 & $\gamma_{1}$ \\
\hline 1 & 0,35 & 0,35 & 0,35 & 0,35 & 0,35 & 0,35 & 0,35 & 0,35 & 0,35 & 0,34 & 0,34 & 0,34 & $-0,10$ \\
2 & 0,60 & 0,20 & 0,19 & 0,28 & 0,35 & 0,38 & 0,40 & 0,40 & 0,40 & 0,40 & 0,38 & 0,34 & $-0,24$ \\
3 & 0,70 & 0,20 & 0,11 & 0,27 & 0,36 & 0,40 & 0,43 & 0,43 & 0,43 & 0,42 & 0,40 & 0,33 & $-0,45$ \\
4 & 0,80 & 0,20 & 0,08 & 0,26 & 0,37 & 0,43 & 0,45 & 0,47 & 0,46 & 0,45 & 0,41 & 0,33 & $-0,61$ \\
\hline
\end{tabular}

Asymmetrie für die realisierten Heritabilitäten auftritt. Für Variante 4 nimmt die realisierte Heritabilität $\left(h_{r}^{2}\right)$ Werte zwischen 0,26 und 0,47 an, wobei $h_{r}^{2}$ für $\alpha$ von 0,1 bis 0,5 wächst und mit weiter steigendem $\alpha$ wieder abfällt. Setzt man für die polygene Heritabilität des heterozygoten Genotyps einen größeren Wert als für die Heritabilität des homozygot positiven Genotyps an, so ergeben sich die in Tabelle 6 aufgelisteten Abhängigkeiten.

Tabelle 6

Realisierte Heritabilităt in Abhăngigkeit von der Remontierung bei $s^{2}=0,2$ (polygene Heritabilităt maximal für heterozygoten Genotyp) (Realized heritability calculated for different selection intensities by $s^{2}=0.2$ )

\begin{tabular}{cccccccccccccc}
\hline \multirow{2}{*}{ Nr.: } & $h_{p_{1}}^{2}$ & $h_{p_{2}}^{2}$ & $h_{p_{3}}^{2}$ & 0,1 & 0,2 & 0,3 & 0,4 & 0,5 & 0,6 & 0,7 & 0,8 & 0,9 & $\gamma_{1}$ \\
\hline 1 & 0,30 & 0,60 & 0,19 & 0,36 & 0,37 & 0,38 & 0,37 & 0,37 & 0,36 & 0,35 & 0,33 & 0,30 & $-0,24$ \\
2 & 0,30 & 0,80 & 0,12 & 0,36 & 0,39 & 0,40 & 0,40 & 0,40 & 0,38 & 0,36 & 0,33 & 0,28 & $-0,42$ \\
3 & 0,40 & 0,60 & 0,05 & 0,41 & 0,42 & 0,42 & 0,42 & 0,42 & 0,41 & 0,40 & 0,38 & 0,33 & $-0,97$ \\
4 & 0,40 & 0,80 & 0,05 & 0,42 & 0,45 & 0,46 & 0,46 & 0,45 & 0,44 & 0,42 & 0,38 & 0,32 & $-1,16$ \\
\hline
\end{tabular}

Die Parameterkonstellation in Tabelle 6 bedingt, daß sich die realisierten Heritabilitäten für Remontierungen zwischen 0,2 und 0,6 annähernd konstant verhalten und für die Remontierungen 0,7, 0,8 und 0,9 ein starker Abfall zu verzeichnen ist. In diesem Sinne ergibt sich eine, zu den Ergebnissen von Tabelle 5, spiegelbildliche Asymmetrie.

3. Schlußfolgerungen

Die Erweiterung des QTL-Anteils von einem auf mehrere Loci bereitet mathematisch keine größeren Schwierigkeiten. Setzt man im Mehr-Locus-Fall Kopplung zwischen den einzelnen Genorten voraus, so wird die Asymmetrie am stärksten ausgeprägt sein, fails alle Loci auf einem Chromosom liegen und zwischen allen Loci zusätzlich vollständige Kopplung besteht. Setzt man Abhängigkeit zwischen genotypischem Wert am QTL und der polygenen Komponente voraus, so wird Kopplung zwischen dem Hauptlocus und den Loci, die Bestandteil der polygenen Komponente sind, impliziert. Vollständig gekoppelte Loci können wieder mit dem QTL identifiziert werden. Aus diesem Grunde wurde das gemischte Modell der Vererbung (GMV) in seiner einfachsten Form zugrunde gelegt.

Die Erzeugung von asymmetrischen Merkmalsverteilungen, wie sie an Daten von Legehennen geschätzt wurden, gelingt mit dem GMV in seiner einfachsten Form, falls 
ein hoher Anteil von QTL-Varianz an der genetischen Gesamtvarianz zugelassen wird. Liegt der Anteil genetischer Varianz an der phänotypischen Varianz bei $40 \%$ und soll der Anteil QTL-Varianz im GMV 20\% nicht übersteigen, so bedarf es zusätzlicher Annahmen über die Umwelt- und polygenen Varianzen am QTL. Sind die Verhältnisse der Umweltvarianzen für die 3 QTL-Genotypen stark ausgelenkt, so bleibt die Merkmalsasymmetrie, hier charakterisiert durch Schiefe und Exzeß, auch unter dem Einfluß von mittel- und langfristiger Selektion auf hohem Niveau erhalten. Merkmalsasymmetrien, hervorgerufen durch unterschiedliche polygene Varianzen werden dagegen bereits nach einer Generation erheblich reduziert, vorausgesetzt zwischen QTL und polygener Komponente besteht keine Abhängigkeit. Asymmetrische Merkmalsverteilungen bewirken, daß die realisierte Heritabilität als Quotient aus Selektionserfolg und Selektionsdifferenz abhängig wird von der Remontierung, d.h. von dem Anteil der selektierten Tiere. Durch gezielte Variation der Umweltvarianzen am QTL können sowohl ein Anstieg als auch ein Abfall für die realisierten Heritabilitäten als Funktion der Remontierungen erklärt werden.

Merkmalsausprägungen, basierend auf dem GMV, sind auch unter dem Einfluß von Selektion über mehrere Generationen dazu geeignet, verschiedene Asymmetrien für die realisierten Heritabilitäten abzubilden. Allerdings bedarf es dazu teilweise extremer Annahmen wie: a) vollständige oder Überdominanz am QTL und b) ein hoher Anteil QTL-Varianz an der phänotypischen Varianz. Soll der Anteil QTL-Varianz an der phänotypischen Varianz gering ausfallen, so muß im GMV Genotyp-Umwelt-Interaktion zugelassen werden.

\footnotetext{
Literatur

BULMER, M.G.:

The effect of selection on genetic variability. The American Naturalist 105 (1971), 201-211

DEKKERS, J.C.M; VAN ARENDONK, J.A.M.:

Optimum selection on identified quantitative trait loci. Proceedings of the $6^{\text {th }}$ World Congress on Genetics Applied to Livestock Production. Armidale Australia, 26 (1998), 361-364

FALCONER, D.S.:

Einfuhrung in die Quantitative Genetik. (Übersetzung von GLODEK, P.) Eugen Ulmer (1984) Stuttgart

HAFNER, R.:

Wahrscheinlichkeitsrechnung und Statistik. Springer-Verlag, Wien New York, (1989)

KOERHUIS, A.N.M.:

Non-normality of egg production distributions in poultry and the effects of outlier elimination and transformation on size and curvilinearity of heritability. Livestock Production Science 45 (1996),69-85

MIELENZ, N.; SCHÜLER, L.:

Auswirkungen eines QTL auf die Asymmetrie der Merkmalsausprägung. Arch. Tierz., Dummerstorf 41(1998) 4, 407-414

MIELENZ, N.:

Berechnung des Selektionserfolges bei Stutzungsselektion in einem gemischten Modell der Vererbung. Unveroffentlichtes Manuskript (2000)

PONG-WONG, R.; WOOLLIAMS, J.A.: Response to mass selection when an identified major gene is segregating. Genet. Sel. Evol. 30 (1998), 313-337

SIMIANER, H.:

Manuskript zum Kurs „Markergestutzte Selektion in der Tierzucht - Kunstliche Selektion unter verschiedenen genetischen Modellen", Universităt Hohenheim 13.-17. November 1995
} 
SAVAS, T.; PREISINGER, R.; RÖHE, R.; THOMSEN, H.; KALM, E.:

The effect of the Box-Cox transformation on the estimation of breeding values for egg production. Proceedings of the $6^{\text {th }}$ World Congress on Genetics Applied to Livestock Production. Armidale Australia, 24 (1998), 353

Eingegangen: 20.07.2000

Akzeptiert: 21.08 .2000

Anschrift der Verfasser

Dr. NORBERT MIELENZ, Univ.-Prof. Dr. habil. LUTZ SCHÜLER

Institut fur Tierzucht und Tierhaltung mit Tierklinik der

Martin-Luther-Universităt Halle-Wittenberg

Adam-Kuckhoff-Straße 35

D-06108 Halle

E-Mail: mielenz@landw.uni-halle.de, schueler@landw.uni-halle.de 